\title{
Collaborative Compressive Sensing based Dynamic Spectrum Sensing and Mobile Primary User Localization in Cognitive Radio Networks
}

\author{
Lanchao Liu and Zhu Han \\ ECE Department \\ University of Houston \\ Houston, Texas 77004
}

\author{
Zhiqiang Wu \\ EE Department \\ Wright State University \\ Dayton, OH 45435
}

\author{
Lijun Qian \\ ECE Department \\ Prairie View A \& M University \\ Prairie View, Texas 774461
}

\begin{abstract}
In wideband cognitive radio (CR) networks, spectrum sensing is one of the key issues that enable the whole network functionality. Collaborative spectrum sensing among the cognitive radio nodes can greatly improve the sensing performance, and is also able to obtain the location information of primary radios (PRs). Most existing work merely studies the cognitive radio networks with static PRs, yet how to deal with the situations for mobile PRs remains less addressed. In this paper, we propose a collaborative compressive sensing based approach to estimate both the power spectrum and locations of the PRs by exploiting the sparsity facts: the relative narrow band nature of the transmitted signals compared with the broad bandwidth of available spectrum and the mobile PRs located sparsely in the operational space. To effectively track mobile PRs, we implement a Kalman filter using the current estimations to update the location information. To handle dynamics in spectrum usage, a dynamic compressive spectrum sensing algorithm is proposed. Joint consideration of the above two techniques is also investigated. Simulation results validate the effectiveness and robustness of the proposed approach.
\end{abstract}

\section{INTRODUCTION}

The rapid development in wireless communication has given rise to a tremendous demand on scarce spectrum resources. Nowadays, most frequency spectrum bands are under-utilized due to the fixed spectrum assignment policy. To increase the spectral efficiency, cognitive radio [1] (CR) has emerged as a promising technology to enable access of unoccupied frequency. One fundamental task of each CR nodes in cognitive radio network is spectrum sensing [2]: detect the presence of the licensed users, also known as the primary radio (PR) nodes, as well as the available unoccupied spectrum. Furthermore, the proliferation of CR devices has fostered the demand for context-aware applications, in which the location information of PRs is viewed as one of the most significant contexts.

In wideband spectrum sensing, CR nodes have to scan multiple frequency bands, which causes long sensing delay or incurs higher computational complexity. Generally, the occupied bands of PRs are often narrow compared to the overall bands scanned. In addition, the active PRs distributed sparsely in the operational area. These spasities in spectrum

This paper is partially supported by NSF ECCS-1028782, CNS-0953377, CNS-0905556, and CNS-0910461. occupation and locations inspire researchers to adopt the recently emerging compressive sensing (CS) [3]-[5] technology, which is now widely utilized in wireless communication [6], to effectively sample the wideband signals. By utilizing the fact that a signal is sparse or compressible in some domain, CS technique can powerfully acquire a signal from a small set of randomly projected measurements with the sampling rate much lower than the Nyquist sampling rate. Then, efficient methods such as basis pursuit (BP) [7], orthogonal matching pursuit (OMP) [8] can be used to reconstruct the original signal. Once the signal is recovered, the spectrum usage and location information of the PRs can be identified.

Exploiting the CS technique, the spectrum sensing and PR localization problem are well studied in [9]-[11]. In [9], the spectrum sensing problem and PR location detection problem are combined together and formulated as a sparse vector. The information of the PRs is reconstructed using the CS technology. In [11] a decentralized way is proposed to solve the spectrum sensing and localization problem. However, existing work mostly investigated the CR network with static PRs. In general, many factors in practice (such as the mobility of PRs and the dynamical spectrum usage) may significantly compromise the detection performance in spectrum sensing. Thus, a more efficient sensing mechanism is needed to handle the dynamics in a practical cognitive radio network.

The main contributions of this paper are as follows. To effectively and efficiently exploit the two-fold sparsity in spectrum and location, we extend our previous work [12] on compressive spectrum sensing to the spectrum sensing and localization problem. To track the location changes of mobile PRs, we implement a Kalman filter to predict the locations of PRs accurately in an intelligent way. Furthermore, dynamic spectrum sensing algorithm is adopted to update the spectrum usage information quickly and reliably. Joint consideration of Kalman filter and dynamic spectrum sensing is also proposed to reduce the searching region and reduce the complexity. Simulation results show efficiency and effectiveness of our proposed approach.

The rest of this paper is organized as follows: The system model of the dynamic spectrum sensing and primary user 
localization problem is given in Section II. Section III details the proposed algorithm in this paper. Section IV illustrates the simulation results and at last we come to the conclusions and future work in Section V.

\section{SySTEM MODEL}

Consider a cognitive radio network with $S$ mobile PRs located sparsely in the operational space as shown in Fig.1. The PRs can move in arbitrary directions in the space with limited velocity, and they stop/start using their licensed spectrum randomly. $N_{r}$ CR nodes in the same field work collaboratively to monitor the dynamic spectrum usage for PRs' transmission as well as the location changes of these PRs.

With regards to the transmitting signals, a slotted frequency segmentation model is adopted, in which the whole bandwidth is divided into $N$ non-overlapping narrowband slots centered at $\left\{f_{v}\right\}_{v=1}^{N}$. Each frequency slot can be viewed as a channel for transmission. The power of the transmitted signal of a PR is expanded as:

$$
P_{s}^{i}=\sum_{v=1}^{N} P_{s}^{i}\left(f_{v}\right),
$$

where $P_{s}^{i}\left(f_{v}\right)$ is the power of the transmitted signal of the $i^{t h}$ PR at the $v^{t h}$ frequency slot, $i=1, \ldots, N_{s}$, and $v=1, \ldots, N$.

Since the transmitted signal will be attenuated according to the distance between the PRs and CRs, in order to estimate the path loss, we assume that any active transmitter locates at a certain finite point out of $N_{s}$ reference points. The channel can be modeled as AWGN and the channel gain is: ${ }^{1}$

$$
H_{i, j}=P_{s}^{i}\left(d_{i, j}\right)^{-\alpha / 2},
$$

where $d_{i j}$ is the distance between the $i^{\text {th }} \mathrm{PR}$ and the $j^{\text {th }} \mathrm{CR}$ and $\alpha$ is the propagation factor. The received power of the transmitted signal at the $j^{t h} \mathrm{CR}$ is expressed as:

$$
P_{r}^{j}\left(f_{v}\right)=\sum_{i=1}^{N_{s}} P_{s}^{i}\left(f_{v}\right) H_{i, j} .
$$

Using the discrete time Fourier transform, $P_{r}^{j}\left(f_{v}\right)$ can be estimated at frequencies $\left\{f_{k}=2 \pi k / N\right\}_{k=0}^{N-1}$ from a number of $N$ time domain samples. To reduce the number of samples, each CR is equipped with a set of frequency selective filters that take the linearly random measurements from all frequency components of the signal. The sensing process at each CR can be represented by an $M \times N$ matrix $\phi$ that randomly maps the signal of length $N$ into $M$ random measurements, where $M \ll N$. The entries of matrix $\phi$ can be designed to be random numbers. The frequency selective filter can be implemented by the frequency-selective surface [13].

At the fusion center, the measurements from $N_{r}$ CRs can be written as an $M N_{r} \times 1$ vector:

$$
\boldsymbol{M}_{M N_{r} \times 1}=\boldsymbol{\Phi}_{M N_{r} \times N N_{r}} \boldsymbol{H}_{N N_{r} \times N N_{S}} \boldsymbol{P}_{\boldsymbol{s}_{N N_{s} \times 1}},
$$

${ }^{1}$ For a fading channel, the channel gain can be calculated by $H_{i, j}=$ $P_{s}^{i}\left(d_{i, j}\right)^{-\alpha / 2}\left|h_{i, j}\right|$ where $h_{i, j}$ is the channel fading gain that can be obtained by averaging out the effect of the channel fading.

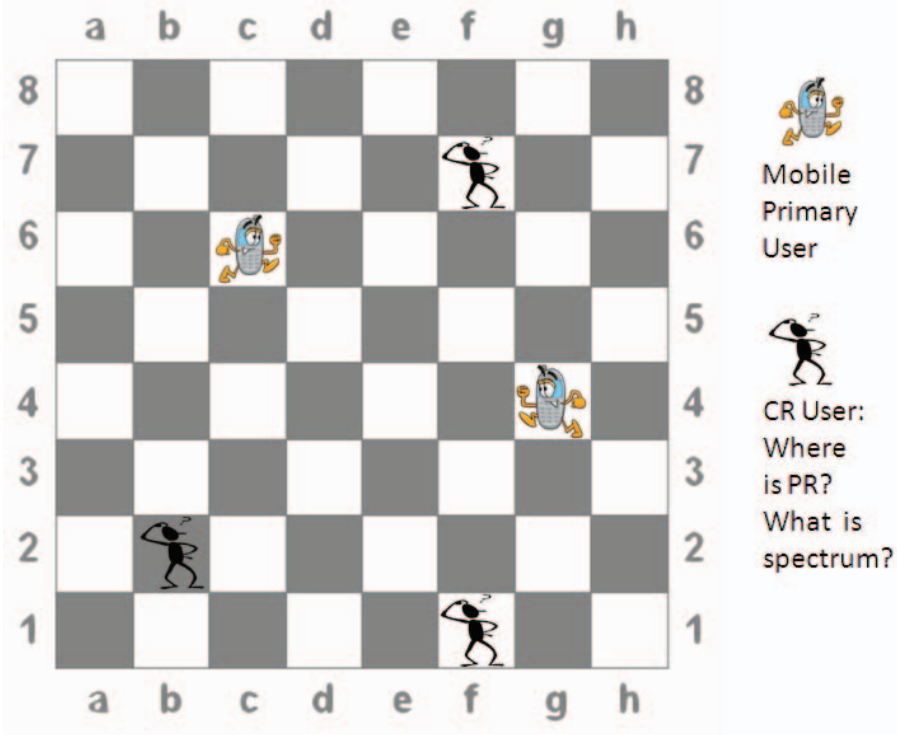

Fig. 1. System Illustration

where $\boldsymbol{P}_{\boldsymbol{s}}$ is a vector with entries:

$$
\boldsymbol{P}_{\boldsymbol{s}}=\left[P_{s}^{1}\left(f_{1}\right), \cdots, P_{s}^{1}\left(f_{N}\right), \cdots, P_{s}^{N_{s}}\left(f_{1}\right), \cdots, P_{s}^{N_{s}} f_{N}\right]^{\top},
$$

and $\boldsymbol{H}$ is a $N N_{r} \times N N_{s}$ matrix:

$$
\mathbf{H}=\left[\begin{array}{cccc}
\bar{H}_{11} & \bar{H}_{12} & \ldots & \bar{H}_{1 N_{s}} \\
\bar{H}_{21} & \bar{H}_{22} & \ldots & \bar{H}_{2 N_{s}} \\
\vdots & \vdots & \ddots & \vdots \\
\bar{H}_{N_{r} 1} & \bar{H}_{N_{r} 2} & \ldots & \bar{H}_{N_{r} N_{s}}
\end{array}\right]
$$

where $\bar{H}_{j i}$ is a diagonal matrix with $H_{j i}$ on its diagonal. $\boldsymbol{\Phi}$ is a $M N_{r} \times N N_{r}$ block diagonal matrix where all of the blocks on the main diagonal are $\phi$ :

$$
\Phi=\left[\begin{array}{cccc}
\phi & 0 & \ldots & 0 \\
0 & \phi & \ldots & \vdots \\
\vdots & \vdots & \ddots & 0 \\
0 & \ldots & 0 & \phi
\end{array}\right]
$$

$\mathbf{0}$ is the matrix whose entries are all zeros.

We rewrite (4) in a more compact form:

$$
\boldsymbol{M}=\boldsymbol{\Phi} H \boldsymbol{P}_{\boldsymbol{s}}=\boldsymbol{A P} \boldsymbol{P}_{\boldsymbol{s}}
$$

In general, we have significantly less measurements $M N_{r} \ll$ $N N_{s}$. Solving this kind of underdetermined linear system of equations is usually time consuming and lowly effective. However, by adopting the recently developed compressive sensing technology, we can delicately recover $\boldsymbol{P}_{\boldsymbol{s}}$ that represents the occupied spectrum and locations of the PRs by exploiting the joint spasity.

In addition, $\boldsymbol{P}_{\boldsymbol{s}}$ may be different at different time instances due to the mobility of the PRs or the dynamic usage of the spectrum. Yet, applying compressive sensing technique to recover $\boldsymbol{P}_{\boldsymbol{s}}$ each time is inefficient. In this paper, we adopt a 
Kalman filter to predict the location change more accurately according to their moving pattern. As to the dynamic spectrum usage situation, the proposed algorithm enables the CR nodes to respond to spectrum change quickly. Furthermore, joint consideration of the Kalman filter and dynamic spectrum sensing is also proposed to reduce the searching region and complexity.

\section{Proposed Algorithm}

In our algorithm, we aim to recover $\boldsymbol{P}_{\boldsymbol{s}}$ to get the spectrum usage and location information of the PRs. Using Kalman filter and DCSS algorithm, $\boldsymbol{P}_{\boldsymbol{s}}$ is constantly updated according to the location change of the PRs and the dynamic spectrum usage environment.

\section{A. Joint Spectrum Sensing and Location}

In (4), $\boldsymbol{M}$ is an $M \times N_{r}$ vector and $\boldsymbol{P}_{\boldsymbol{s}}$ is an $N \times N_{s}$ vector. Since $M \times N_{r} \ll N \times N_{s}$, directly solve the ill-posed problem is time consuming. Notice that the transmitted signals possess a narrow band nature compared with the broad bandwidth of available spectrum as well as the mobile PRs located sparsely in the operational space. Due to these sparsity, most of the entries of $\boldsymbol{P}_{\boldsymbol{s}}$ are zeros. To recover it, we can convert the original ill-posed problem to a convex optimization problem, and apply the $\ell_{1}$ norm minimization:

$$
\min \left\|\boldsymbol{P}_{\boldsymbol{s}}\right\|_{1} \quad \text { s.t. } \quad \boldsymbol{M}=\boldsymbol{A} \boldsymbol{P}_{\boldsymbol{s}} .
$$

We adopt YALL1 [14] to recover $\boldsymbol{P}_{\boldsymbol{s}}$, thus indentify the spectrum usage and localization information of the PRs in the CR network.

\section{B. Kalman Filter for Localization}

To predict the locations of the mobile users more accurate, we implement a Kalman filter [15] to track the location change of the PRs.

At $t=t_{0}, \boldsymbol{P}_{\boldsymbol{s}}$ is recovered from the measurements of the CRs. From $t>t_{0}$, a Kalman filter is used to update $\boldsymbol{P}_{\boldsymbol{s}}$ instead of using $\ell_{1}$ at every time instance. The Kalman filter prediction and update procedure is shown in Fig.2.

The input state $\boldsymbol{X}$ of the Kalman filter is defined as $\boldsymbol{X}=$ $\left[\boldsymbol{P}_{\boldsymbol{s}}, \widetilde{\boldsymbol{L}_{s}}\right]^{\top}$, where $\widetilde{\boldsymbol{L}_{s}}$ is the location change information from the PRs inertial navigation systems. We predict the next state as:

$$
\boldsymbol{X}_{t \mid t-1}=\boldsymbol{F} \boldsymbol{X}_{t-1},
$$

where $\boldsymbol{F}$ is the state transition matrix:

$$
\mathbf{F}=\left[\begin{array}{ll}
1 & 1 \\
0 & 1
\end{array}\right]
$$

$\mathbf{1}$ is an $N N_{s}$ vector consist of ones and $\mathbf{0}$ is an $N N_{s}$ vector consist of zeros.

Then, the prediction is updated with the measurement innovation with Kalman filter gain:

$$
\boldsymbol{X}_{t}=\boldsymbol{X}_{t \mid t-1}+\boldsymbol{K}_{t}\left(\overline{\boldsymbol{M}}_{t}-\overline{\boldsymbol{A}} \boldsymbol{X}_{t \mid t-1}\right),
$$

where $\overline{\boldsymbol{M}}_{t}$ is a $2 M N_{r}$ measurement vector $\overline{\boldsymbol{M}}_{t}=[\boldsymbol{M}, \mathbf{0}]^{\top}$. $\overline{\boldsymbol{A}}$ is the $2 M N_{r} \times N N_{s}$ measurement matrix $\overline{\boldsymbol{A}}=[\boldsymbol{A} ; \mathbf{0}]$.

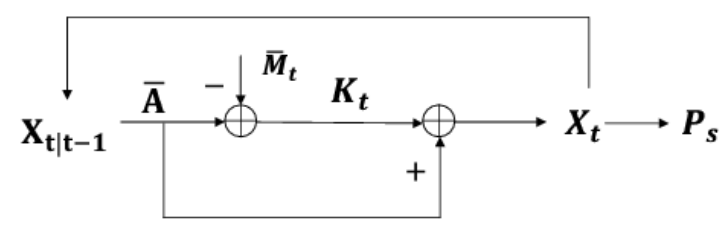

Fig. 2. Localization using Kalman filter. $\overline{\boldsymbol{A}}_{t}$ is the measurement matrix. $\boldsymbol{K}^{t}$ is the Kalman filter gain updated at every time instance. $\overline{\boldsymbol{M}}_{t}$ is the measurements at the fusion center at every time instance.

$\left(\overline{\boldsymbol{M}}_{t}-\overline{\boldsymbol{A}} \boldsymbol{X}_{t \mid t-1}\right)$ is the measurement innovation. $\boldsymbol{K}^{t}$ is the Kalman filter gain which is updated at every time instance:

$$
\boldsymbol{K}_{t}=\boldsymbol{E}_{t \mid t-1} \overline{\boldsymbol{A}}^{\top}\left(\overline{\boldsymbol{A}} \boldsymbol{E}_{t \mid t-1} \overline{\boldsymbol{A}}^{\top}+\boldsymbol{R}\right)^{-1},
$$

where

$$
\boldsymbol{E}_{t \mid t-1}=\boldsymbol{F} \boldsymbol{E}_{t-1} \boldsymbol{F}^{\top}+\boldsymbol{Q},
$$

$\boldsymbol{R}$ is the measurement noise covariance matrix, and $\boldsymbol{Q}$ is the prediction process noise covariance matrix, which can both be obtained from off-line measurements. $\boldsymbol{E}$ is the priori estimate error covariance matrix updated at each iteration by the following formula:

$$
\boldsymbol{E}_{t}=\left(\mathbf{1}-\boldsymbol{K}_{t} \overline{\boldsymbol{A}}\right) \boldsymbol{E}_{t \mid t-1} .
$$

From state $\boldsymbol{X}$ at time $t$, we can obtain $\boldsymbol{P}_{\boldsymbol{s}}$ and find out the locations of the PRs.

\section{Dynamic Spectrum Sensing}

At every time instance, we calculate the measurement difference $\Delta \boldsymbol{M}=\left|\boldsymbol{M}_{t_{1}}\right|-\left|\boldsymbol{M}_{t_{2}}\right| . t_{1}$ and $t_{2}$ denote two different time instances. Once $|\Delta M|$ exceeds the preselected threshold $\tau$, it means that the spectrum occupation of the CR network is varied. Either an existing PR stops or starts using a channel, or a new PR joins the network in this situation. To deal with the dynamics in spectrum usage, we adopt dynamic compressive spectrum sensing to solve it as a least-squares problem:

$$
\min _{n \in\left\{1, \ldots, N_{s} N\right\}} \min _{\left(P_{s}\right)_{n}}\left\|\boldsymbol{A}_{n}\left(P_{s}\right)_{n}-\Delta \boldsymbol{M}\right\|_{2},
$$

where $\boldsymbol{A}_{n}$ is the $n^{\text {th }}$ column of sensing matrix $\boldsymbol{A}$, and $\left(P_{s}\right)_{n}$ is the $n^{t h}$ entity in vector $\boldsymbol{P}_{\boldsymbol{s}}$.

In the case of an existing PR release its occupied channel, the norm of $M_{t_{2}}$ is less than that of $M_{t_{1}}$. Using the location estimation results of the Kalman filter, we can find out the changing entry $\left(P_{s}\right)_{n}^{*}$ by searching in a reduced region $\Omega$ :

$$
\min _{n \in \Omega}\left\|\boldsymbol{A}_{n}\left(\boldsymbol{P}_{\boldsymbol{s}}\right)_{n}^{*}-\Delta \boldsymbol{M}\right\|_{2}, \quad n=1, \ldots, S .
$$

where $\Omega$ denote the set of entries that represent the identified active PRs.

For the situation of a new PR joining the existing CR network, the minimizer of objective function (12) can be written as

$$
\left(\boldsymbol{P}_{\boldsymbol{s}}\right)_{n}^{*}=\frac{\left(\boldsymbol{A}_{n}\right)^{\top}(\Delta \boldsymbol{M})}{\left(\boldsymbol{A}_{n}\right)^{\top} \boldsymbol{A}_{n}}, \quad n=1, \ldots, N N_{s} .
$$


We can use a vectorized algorithm [16] to get $\left(P_{s}\right)_{n}^{*}$. Then compare

$$
\left\|\boldsymbol{A}_{n}\left(P_{s}\right)_{n}^{*}-\Delta \boldsymbol{M}\right\|_{2}, n=1, \ldots, N N_{s} .
$$

If there is a unique $n$ that gives 0 or a tiny value, then this $n$ is $n^{*}$, the entity that has changed since the previous period. Therefore, let

$$
\boldsymbol{P}_{\boldsymbol{s}} \leftarrow\left(\boldsymbol{P}_{\boldsymbol{s}}\right)_{\text {prev }}+\left\{\begin{array}{ll}
\left(P_{s}\right)_{n}^{*}, & n=n^{*} ; \\
0, & \text { otherwise, }
\end{array} \quad n=1, \ldots, N N_{s} .\right.
$$

Hence, we can detect the change in spectrum usage quickly with high reliability. Once the $\boldsymbol{P}_{\boldsymbol{s}}$ is updated, as a byproduct, we can get the location of the newly present and vanishing $\mathrm{PR}$ in the CR network as well.

Overall, we adopt a mechanism that keeps a balance between the accuracy and the efficiency. To complete the dynamic spectrum sensing and mobile PRs localization, we recover $\boldsymbol{P}_{\boldsymbol{s}}$ using compressive sensing at regular intervals. Between the two reconstructions, $\boldsymbol{P}_{\boldsymbol{s}}$ is constantly updated through Kalman filter predictions and dynamic compressive spectrum sensing results. Thus we can successfully monitor the dynamics of the PRs locations and spectrum usage in the CR network.

\section{Simulation Results}

The simulation setup is described as follows. The operational space is a $1000 \mathrm{~m} \times 1000 \mathrm{~m}$ square field, which contains $N_{s}=100$ uniformly distributed reference points. We monitor the dynamic spectrum usage and the locations of PRs for a time interval $T$. The active primary users randomly locate in the space and move in arbitrary directions in the space, or stop/start using their channels at any time. The time instance $t_{\text {stop }}$ and $t_{\text {start }}$ are selected according to a uniform distribution $U(0, T)$. We use a simple symmetric lattice random walk model to simulate the mobility of the PRs. Thus at each time instance, the probabilities of a PR walk to any one of its neighbors are the identical. The transmitted power of PRs is 1W. The number of available channels $N$ is 64 , and $N_{r}=20$ $\mathrm{CR}$ nodes are randomly deployed in the same field working collaboratively to implement the dynamic sensing, localization and tracking tasks.

To demonstrate the performance of our algorithms, we define the sampling rate $r$ as $r=\left(M \times N_{r}\right) /\left(N \times N_{s}\right)$. The Probability of Detection (POD) and Miss Detection Rate (MDR) are, respectively,

$$
\begin{gathered}
P O D=\frac{N_{H i t}}{N_{H i t}+N_{\text {Miss }}}, \\
M D R=\frac{N_{\text {Miss }}}{N_{\text {Miss }}+N_{\text {Correct }}} .
\end{gathered}
$$

Here, $N_{H i t}$ is the number of successful detections of PRs. $N_{\text {Miss }}$ is the number of miss detections, and $N_{\text {correct }}$ is the number of correct reports of no appearance of PRs.

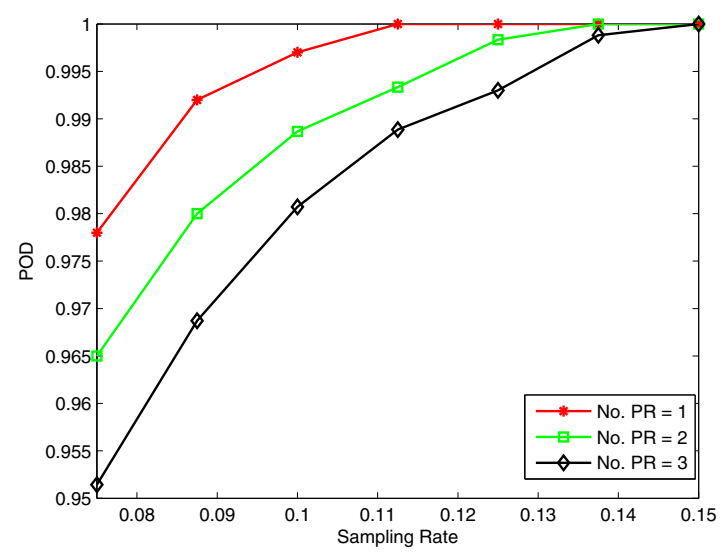

Fig. 3. POD vs. Sampling Rate

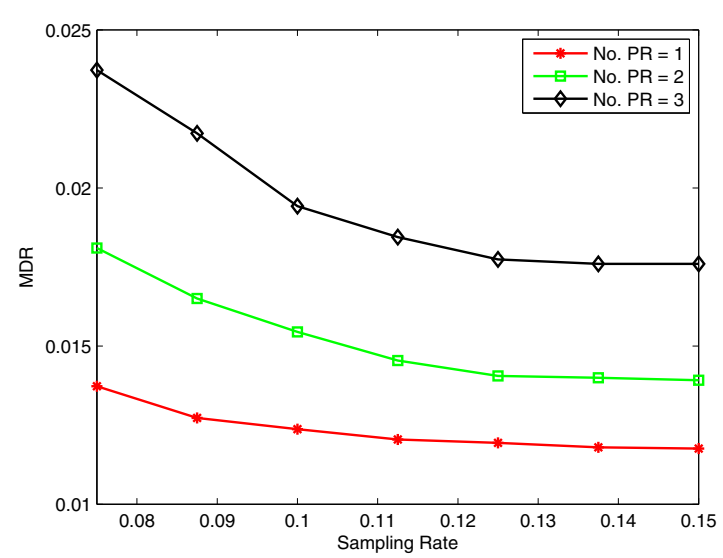

Fig. 4. MDR vs. Sampling Rate

\section{A. Joint Compressive Spectrum Sensing and Localization}

We validate the proposed joint compressive spectrum sensing and localization algorithm. The number of active PRs ranges from 1 to 3 in the simulation and the sampling rate is from $7.5 \%$ to $15 \%$. Fig. 3. shows the probability of detection and Fig. 4. shows the miss detection rate.

In Fig.3, when the sampling rate is $7.5 \%$, the probability of detection is above $95 \%$ in all cases. As the sampling rate increases beyond $15 \%$, spectrum occupation and location information can be recovered with a very high probability. In Fig.4, the miss detection probability is very low at a low sampling rate in all the three cases. The MDR decreases as the increment of the sampling rate. Even when three active PRs appear in the operational space, the MDR can be lower than $2 \%$ as the sampling rate approaches $15 \%$.

\section{B. Kalman Filter Based Location}

Once $\boldsymbol{P}_{\boldsymbol{s}}$ is reconstructed, the location change of the PRs are tracked by applying the Kalman filter at the fusion center. In the operational space, we define the location change from one reference point to another as a step. Without loss of generality, we define the velocity of the PR is one step between two time instances. We investigate the performance of Kalman filter, 


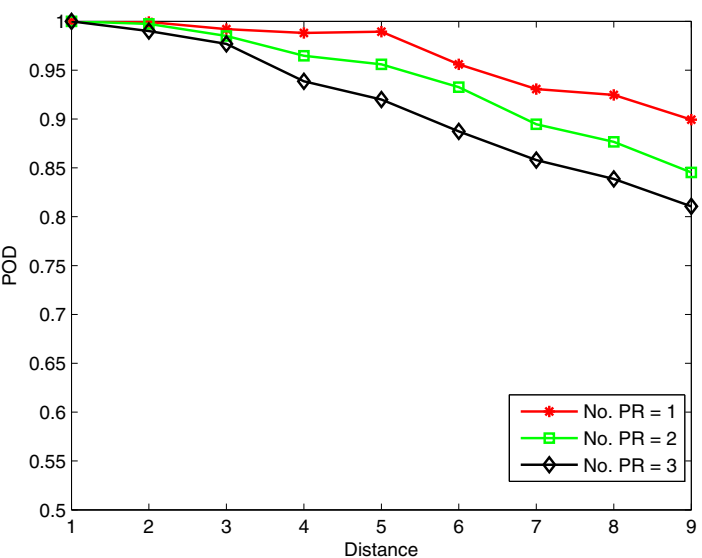

Fig. 5. POD vs. Distance
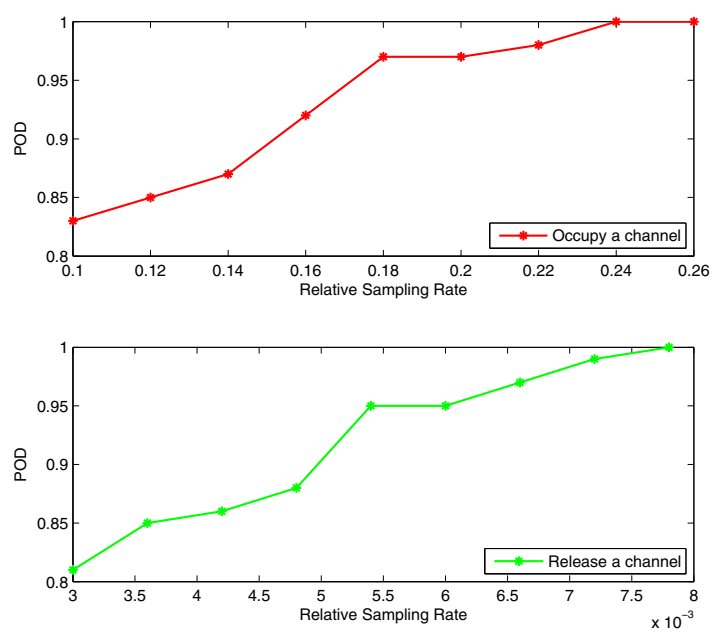

Fig. 6. POD vs. Relative Sampling Rate

using the averaging distance in the operational field of nine steps. Fig. 5 shows the POD as the change of the distance.

We can see that the Kalman filter works effectively when there is only one active PR in the operational field. The POD is above $90 \%$ when walking a trace of nine steps. The probability of detection decreases as the distance increases. When more active PRs appear in the field, the tracking task becomes more challenging. In the worst case, the performance is still acceptable when all three PRs travel the distance of nine steps.

\section{Dynamic Spectrum Sensing}

In Fig.6, we show the probability of detection in dynamic spectrum sensing versus the relative sampling rate. The relative sampling rate is defined as the number of received entries of $\Delta M$ over its total number of entries. Remark that the relative sampling rate is referred to the size of $\Delta M$, which is already much compressed compared to $\boldsymbol{P}_{\boldsymbol{s}}$.

When a new PR occupies a channel, we test the performance of the dynamic spectrum sensing when the relative sampling rate is varied from $10 \%$ to $26 \%$. We can see that as the relative sampling rate increases, the POD approaches one. We can see that at the fusion center, only $26 \%$ entries of $\Delta M$ is needed to guarantee a successful detection. In the case of one existing PR releasing a channel, we can see that the relative sampling rate is quite low. A sampling rate of mere $0.8 \%$ will guarantee a perfect detection.

\section{Conclusions}

In this paper, we exploit the two-fold sparsity in spectrum and location to deal with the spectrum sensing and localization problem in CR networks. To handle the mobility of the PRs and dynamics of the spectrum usage, we implement a Kalman filter to track the location changes of the mobile PRs in an intelligent way, and the proposed spectrum sensing algorithm is applied to update the spectrum usage information quickly and reliably. In addition, joint concern of Kalman filter and dynamic spectrum sensing is also investigated. Simulation results validate the effectiveness of our algorithm.

\section{REFERENCES}

[1] E. Hossain, D. Niyato, and Z. Han, Dynamic spectrum access in cognitive radio networks, Cambridge University Press, 2009.

[2] I. F. Akyildiz, B. F. Lo, and R. Balakrishnan, "Cooperative spectrum sensing in cognitive radio networks: a survey," Physical Communication (Elsevier) Journal, Vol. 4, No. 1, pp. 40-62, Mar. 2011.

[3] D. Donoho, "Compressed sensing," IEEE Transactions on Information Theory, Vol. 52, No. 4, pp. 1289-1306, Apr. 2006.

[4] E. Candes, and T. Tao, "Near optimal signal recovery from random projections: Universal encoding strategies? "IEEE Trans. on Information Theory, Vol. 52, No. 12, pp. 5406-5425, Dec. 2006.

[5] R. Baraniuk, "Compressive sensing," IEEE Signal Processing Magazine, Vol. 2, Iss. 4, pp. 118-121, Jul. 2007.

[6] Y. Li, Z. Han, H. Li, and W. Yin, Compressive Sensing for Wireless Networks, contract with Cambridge University Press, 2012.

[7] S. S. Chen, D. L. Donoho, M. A. Saunders, "Atomic decomposition by basis pursuit," SIAM Journal on Scientific Computing, Vol. 20, No. 1, pp. 33C61, 1998

[8] J. Tropp, A. Gilbert, "Signal recovery from random measurements via orthogonalmatching pursuit," IEEE Transactions on Information Theory, Vol. 53, Iss. 12, pp.4655-4666, Dec. 2007.

[9] X. Li, V. Chakravarthy, and Z. Wu, "Joint Spectrum Sensing and Primary User Localization for Cognitive Radio via Compressed Sensing," in Proc. of IEEE MILCOM, San Jose, California, Oct. 2010.

[10] F. Zeng, C. Li, and Z. Tian, "Distributed compressive spectrum sensing in cooperative multihop cognitive networks," IEEE Journal of Selected Topics in Signal Processing, Vol. 5, Iss. 1, pp.37, Feb. 2010.

[11] J. -A. Bazerque and G. B. Giannakis, "Distributed spectrum sensing for cognitive radio networks by exploiting sparsity," IEEE Transations on Signal Processing, Vol. 58, Iss. 3, pp. 1847-1862, Mar. 2010.

[12] J. Meng, W. Yin, H. Li, E. Hossain, and Z. Han, "Collaborative Spectrum Sensing from Sparse Observations in Cognitive Radio Networks," IEEE Journal on Selected Topics on Communications, special issue on Advances in Cognitive Radio Networking and Communications, Vol. 29, Iss. 2, pp. 327 - 337, Feb. 2011.

[13] B. A. Munk, Frequency Selective Surfaces: Theory and Design, Wiley, 2000.

[14] Y. Zhang, J. Yang, and W. Yin, "YALL1: your algo-rithm for L1," http://yall1.blogs.rice.edu/, 2009

[15] N. Vaswani, "Kalman filtered compressed sensing," in proceedings of International Conference on Image Processing, San Diego, California, Oct. 2008.

[16] W. Yin, Z. Wen, S. Li, J. Meng, and Z. Han, "Dynamic Compressive Spectrum Sensing for Cognitive Radio Networks", in procedings of the 45th Annual Conference on Information Sciences and Systems (CISS), Johns Hopkins University, Mar. 2011. 